\title{
Liame
}

Histoire et histoire de l'art des époques moderne et contemporaine de l'Europe méditerranéenne et de ses périphéries

25 | 2012

Les réseaux négociants dans la France méridionale (XVIII ${ }^{\mathrm{e}}$-XIX ${ }^{\mathrm{e}}$ siècles)

\section{Des réseaux marchands aux consommateurs : la diffusion des indiennes en Europe méditerranéenne dans la seconde moitié du XVII siècle}

\section{Olivier Raveux}

\section{OpenEdition}

\section{Journals}

\section{Édition électronique}

URL : http://journals.openedition.org/liame/227

DOI : 10.4000/liame.227

ISSN : 2264-623X

Éditeur

CRISES - Centre de Recherches Interdisciplinaires en Sciences Humaines et Sociales de Montpellier

Référence électronique

Olivier Raveux, « Des réseaux marchands aux consommateurs : la diffusion des indiennes en Europe méditerranéenne dans la seconde moitié du XVII e siècle », Liame [En ligne], 25 | 2012, mis en ligne le 05 décembre 2012, consulté le 19 avril 2019. URL : http://journals.openedition.org/liame/227 ; DOI : 10.4000/liame.227

Ce document a été généré automatiquement le 19 avril 2019

\section{cc) (†) $\Theta$}

Les contenus de Liame sont mis à disposition selon les termes de la Licence Creative Commons Attribution - Pas d'Utilisation Commerciale - Pas de Modification 4.0 International. 


\title{
Des réseaux marchands aux
} consommateurs : la diffusion des indiennes en Europe méditerranéenne dans la seconde moitié du XVII siècle

\author{
Olivier Raveux
}

1 Consommée à l'échelle planétaire dès la fin du $\mathrm{XvII}^{\mathrm{e}}$ siècle, l'indienne est le premier produit manufacturé des débuts de la mondialisation. C'est à partir des années 1650 que le commerce de ces cotonnades fabriquées en Asie prend son essor sur le vieux continent. En apportant les avantages d'une fibre textile peu utilisée jusqu'alors et en offrant de nouveaux répertoires de décoration, l'indienne finit par transformer la culture matérielle européenne, pour devenir au XVIII ${ }^{e}$ siècle le principal levier du démarrage de la première révolution industrielle ${ }^{1}$. L'Europe méditerranéenne a pleinement participé à cette histoire. Des tissus comme les chafarcanis et les mezzari rappellent d'ailleurs avec force l'appropriation de ces toiles asiatiques par les Provençaux et les Liguriens, qui en ont fait des éléments emblématiques de leur vestiaire et de leur industrie jusqu'au xIx ${ }^{\mathrm{e}}$ siècle.

2 L'indienne a fait son apparition dans le Sud de l'Europe dès les débuts du XVI ${ }^{e}$ siècle mais reste longtemps un achat de curiosité pour quelques personnes fortunées. Tout change à partir de 1650. En quelques décennies, l'indienne cesse d'être un produit rare, onéreux et exotique pour devenir un article à la mode en Italie, en France et en Espagne. Cet article entend décrire ce tournant, en présentant les produits, les routes, les réseaux marchands et les modèles de consommation qui ont permis à l'indienne de s'enraciner en Méditerranée. Le tableau sera partiel. Si l'histoire des indiennes a suscité de nombreuses recherches pour les $\mathrm{XVIII}^{\mathrm{e}}$ et $\mathrm{XIX}^{\mathrm{e}}$ siècles, le XVII ${ }^{\mathrm{e}}$ reste mal connu. Les travaux disponibles et les documents d'archives trouvés ici et là permettent néanmoins de dresser un état des lieux. 


\section{Les indiennes à l'assaut de l'Europe}

3 Pour comprendre la diffusion des indiennes dans la Méditerranée de la seconde moitié du XVII ${ }^{e}$ siècle, il faut tout d'abord s'intéresser à l'histoire générale de l'exportation de ces toiles asiatiques vers l'Europe. Qu'elle touche aux produits, aux routes empruntées ou aux réseaux mobilisés par ce commerce, cette histoire est marquée du sceau de la diversité. Cotonnades indiennes, persanes et ottomanes, marchands orientaux et européens, routes océaniques et caravanes terrestres composent un monde commercial aux ressources multiples.

\section{Routes océaniques et grandes compagnies}

Les indiennes sont acheminées depuis l'Asie par deux routes distinctes. La première, ouverte par Vasco de Gama en 1498, est transocéanique et permet d'apporter les cotonnades de l'Inde par le cap de Bonne-Espérance. C'est la route des compagnies européennes des Indes orientales. Au cours de la seconde moitié du XvII siècle, l'East India Company (EIC), la Verenidge Oost-indische Compagnie (VOC) et la Compagnie française des Indes orientales, fondées respectivement en 1600, 1602 et 1664, acheminent des volumes croissants d'indiennes. L'accélération se situe entre 1665 et 1684, époque durant laquelle les exportations vers l'Europe sont presque multipliées par cinq pour approcher les 1.300 .000 pièces par $\mathrm{an}^{2}$. Ces produits servent d'abord à alimenter les marchés nationaux, avant d'être réexportés vers d'autres régions européennes. Dans la redistribution de ces toiles, l'Angleterre a montré l'exemple. Entre les années 1660 et les années 1699-1701, son commerce annuel de réexportation passe d'une quantité négligeable à 340.000 livres sterling 3 .

Quels sont ces tissus indiens et d'où viennent-ils ? Les archives livrent une grande variété de noms, à l'image des inventaires de magasins textiles de Gênes et Marseille pour les années 1670-16804. Calankars, casses, chafarcanis, lisats, percales, serongis, varas, etc. composent une liste fournie, dans laquelle il est parfois difficile de distinguer les toiles blanches de celles peintes ou imprimées. Toutes proviennent des trois grands centres de l'industrie cotonnière indienne ${ }^{5}$. Le plus ancien et longtemps le plus important est celui du Centre-Ouest et du Nord-Ouest de la péninsule, avec les productions renommées d'Ahmedabad et de Sironj (districts de Mâlwâ et du Gujarat). La suprématie de cette zone reste incontestée jusqu'au milieu des années $1680^{6}$. À la fin du XVII siècle, les productions de la côte est (côte de Coromandel et Bengale) finissent par prendre le dessus pour les expéditions vers le vieux continent.

\section{Routes caravanières et négociants orientaux}

6 La deuxième voie empruntée par les cotonnades de l'Inde au XVII ${ }^{e}$ siècle se greffe en partie sur la vieille route de la soie. Les indiennes sont chargées dans l'océan Indien, à Surat, gagnent la Perse, traversent le continent asiatique et parviennent en Europe en traversant la Méditerranée. Les caravanes intra-asiatiques partent de Bandar-Abbas et aboutissent à Alep et à Smyrne, les deux principales échelles ottomanes ${ }^{7}$.

7 Les indiennes de l'Inde sont-elles les seules cotonnades asiatiques à gagner l'Europe par les Échelles du Levant? L'impression sur coton est aussi l'affaire des Perses et des 
Ottomans. Chemin faisant entre océan Indien et Méditerranée, la gamme des produits destinés à l'exportation vers l'Europe s'élargit. Les caravanes prennent en charge d'autres cotonnades comme les toiles "peinture de Perse», les boucassins de Smyrne ou les chafarcanis d'Alep. Que valent ces tissus par rapport aux véritables indiennes? Une règle prévaut alors: la qualité des indiennes asiatiques se dégrade au fur et à mesure de l'avancée vers l'ouest. Plusieurs facteurs expliquent cette situation comme la qualité des produits employés ou le coût des compétences techniques et artistiques de la maind'œuvre. L'authentique indienne n'a pas vraiment de rivale et les productions ottomanes sont d'ailleurs surtout destinées aux gens du peuple. L'indienne de Perse occupe quant à elle un rang intermédiaire. Proche des productions ottomanes quand elle est fabriquée pour le marché intérieur persan, elle devient un tissu de qualité quand elle est destinée à l'exportation ${ }^{8}$. L'indienne persane exportée vers les États du Grand Seigneur ou vers l'Europe, le plus souvent imprimée dans les ateliers d'Ispahan, est produite à partir d'une toile blanche des Indes. Ce sont, par exemple, ces toiles lisats "peinture de Perse" acheminées par le négociant arménien Raphaël Ruply à la foire de Beaucaire en $1675^{9}$. Certains centres de l'Empire ottoman peuvent agir de la même manière et l'on trouve parfois dans les boutiques des « lisats peints à Smyrne $»^{10}$. La pratique est plus limitée et, de toute manière, l'écart de qualité entre l'Empire ottoman et la Perse dans l'art des miniatures se retrouve dans la fabrication des indiennes.

Les Européens n'ont aucun rôle dans l'acheminement de ces toiles à travers l'Asie. Les Orientaux en ont le monopole et nombreux sont les groupes marchands à pratiquer cette activité : Perses, Turcs, Arabes, minoritaires comme les Juifs, les Grecs, les Maronites et les Arméniens. Ce sont ces derniers qui dominent le commerce intra-asiatique des indiennes au XVII ${ }^{\mathrm{e}}$ siècle. Plusieurs éléments ont joué à leur avantage. Chrétiens et donc neutres dans les conflits entre sunnites ottomans et chiites persans, ils tirent profit de l'expérience accumulée dans l'exportation des soies persanes et de la position de l'Arménie sur les routes caravanières. Un groupe en particulier se distingue, celui de la Nouvelle-Djoulfa, une banlieue d'Ispahan, capitale de la Perse safavide. Présents de Canton à Cadix, ces Arméniens de Perse ont tissé un réseau mondial de circulation d'agents, d'informations et de produits et sont devenus les intermédiaires obligés du négoce eurasiatique ${ }^{11}$. Si la soie est leur produit de prédilection, l'indienne fait également leur richesse et ils ne se limitent pas à la faire voyager. Les Djoulfains ont agi comme marchands-fabricants. Ils ont suscité et orienté la production d'indiennes dans les places de commerce où ils travaillaient. Ainsi, afin d'être plus efficaces sur les marchés persans, ottomans et européens, les négociants arméniens de la Nouvelle-Djoulfa faisaient travailler à façon les imprimeurs de l'Inde moghole ou importaient des toiles blanches des Indes, pour les faire travailler par des artisans d'Ispahan ${ }^{12}$.

\section{Les raisons du succès}

Par la combinaison des deux routes et par la diversité des réseaux marchands, l'arrivée des indiennes dans l'Europe de la seconde moitié $d u$ XVII ${ }^{\mathrm{e}}$ siècle devient massive. Ces toiles sont désormais disponibles dans une gamme ouverte de modèles et de prix, susceptible de toucher un éventail de consommateurs allant du monde des artisans aux élites. Deux raisons expliquent ce succès.

10 Au moment de leur fondation, les compagnies des Indes cherchaient à écouler en Asie des produits manufacturés européens, comme les draps de laine, et à en ramener les fameux 
épices. Quand elles s'intéressaient aux indiennes, c'était dans le cadre d'échanges intraasiatiques. La donne évolue à partir des années 1650-1670, quand les exportations d'épices vers l'Europe peinent à poursuivre leur croissance ${ }^{13}$. Maîtres des îles à épices, les Hollandais avaient fait le choix d'une politique de production malthusienne, afin de conserver des taux de profits élevés. Conséquences? Ce commerce ne porte plus la progression du chiffre d'affaires des compagnies européennes. Un nouveau produit devait prendre le relais et la cotonnade s'est alors imposée comme la principale marchandise d'exportation vers l'Europe ${ }^{14}$.

11 Amener ces cotonnades en Europe était une chose. Les vendre en était une autre. Jusqu'aux années 1650 , à cause de ses fonds colorés appuyés et de ses motifs décoratifs culturellement éloignés, l'indienne ne trouve pas sa place dans la consommation textile européenne ${ }^{15}$. Si la situation change, c'est sous l'effet conjugué des politiques menées par les compagnies des Indes pour mettre en place une production asiatique adaptée aux goûts européens et de la capacité des artisans indiens à modifier leur fabrication. Il faut rappeler ici le rôle précurseur de l'EIC ${ }^{16}$. Les Anglais entament leur stratégie d'ajustement dès 1643 , quand les directeurs de la compagnie demandent à leurs agents en Inde de prendre des toiles dont les motifs s'accordent le mieux aux goûts des consommateurs britanniques. En 1662, une nouvelle étape est franchie avec l'envoi d'échantillons pour qu'ils soient copiés sur place. Enfin, durant les années 1670-1680, ce sont des artisans britanniques qui sont dépêchés en Asie avec leurs matériels de production, pour former les artisans indiens aux attentes des consommateurs occidentaux. Au final, l'exotisme est devenu façade. L'indienne achetée par les Européens est consommée comme un pur produit asiatique alors qu'elle est le plus souvent fabriquée pour eux seuls. Les réseaux marchands arméniens ont également pris part à cette transformation des productions asiatiques et nous avons souligné plus haut le rôle des négociants de la Nouvelle-Djoulfa en ce domaine. En revanche, nous mesurons encore mal l'impact du commerce privé européen dans les Échelles du Levant. Il a pu être important comme en témoigne l'ouverture d'une fabrique d'indiennes à Smyrne par le Marseillais Pierre Chaulier dans les années $1680^{17}$.

\section{Accueil, distribution et consommation sur les rives nord-méditerranéennes}

12 Arrivées dans le Nord de l'Europe ou dans les Échelles du Levant, les cotonnades asiatiques sont prises en charge par de nouveaux réseaux marchands pour atteindre les ports méditerranéens de l'Espagne, de la France et de l'Italie. Ces importations et ces redistributions s'appuient sur une hiérarchie portuaire dominée par Livourne et Marseille.

\section{Réseaux et circuits d'importation et de redistribution}

$13 \mathrm{Au} \mathrm{XVII}^{\mathrm{e}}$ siècle, la réexpédition des indiennes depuis le Nord de l'Europe s'appuie sur les réseaux des correspondants de l'EIC et de la VOC. Les deux compagnies sont solidement implantées en Italie, en Espagne et dans le Sud de la France ${ }^{18}$. Un seul chiffre suffit pour montrer la vigueur de leur activité : en 1686, elles envoient plus de 50.000 pièces de coton des Indes vers Marseille ${ }^{19}$. 

XVII ${ }^{e}$ siècle. Durant les années 1650-1700, Harry Brown, Thomas Dethick, Robert Foot, Gilbert Searle ou encore Humphrey Sidney ont fait du port franc toscan «the scale or magazine of an universal English trade $»^{20}$ et une plateforme de redistribution pour les indiennes expédiées depuis Londres. Pour la même période, ils sont également nombreux à tenir «maison et raison en la ville de Marseille » (John Goodwin, Robert Lang, John Launce, Robert Shaw et Thomas Warren). Deux figures se détachent dans ce groupe. John Launce est l'agent local de l'EIC dans les années 1660 et paraît avoir été le premier à vendre localement les indiennes envoyées d'outre-Manche ${ }^{21}$. Robert Lang lui succède dans cette tâche dans les années 1670-168022. Naturalisé français et consul d'Angleterre, il est un des grands pourvoyeurs locaux de tissus asiatiques ${ }^{23}$. D'autres villes portuaires pourraient être ajoutées sans peine: là où les débouchés pour les indiennes sont importants, les marchands anglais sont présents.

Les Hollandais ne sont pas en reste. Autour de la figure de Nicolas Rutz, ils ont développé une présence active à Livourne et à Marseille. Ce négociant originaire d'Amsterdam, nommé consul de Hollande dans les deux ports, est le grand représentant des Bataves dans la Méditerranée des années 1670-1680 et y réceptionne les produits de la VOC ${ }^{24}$. Les liaisons commerciales entre les Pays-Bas et la Méditerranée sont plus complexes que celles développées par l'Angleterre car elles sont également animées par des marchands italiens implantés à Amsterdam. Les travaux d'Antonella Bicci ont montré comment, durant les années 1670-1680, la société amstellodamoise du marchand juif italien Beniamino Burlamacchi irriguait, par le biais de nombreux correspondants, les villes portuaires de l'Italie centrale et les Etats pontificaux de produits divers dont les « tele di cotone stampate » de la $\mathrm{VOC}^{25}$.

16 Le panorama des exportations d'indiennes depuis les Échelles du Levant est bien différent. Nous retrouvons certes les marchands anglais et hollandais mais l'éventail des nationalités et des communautés marchandes s'ouvre considérablement, comme le montre le rapport du Français d'Ortières pour le commerce d'Alep en $1686^{26}$. Il y a tout d'abord les nations engagées depuis longtemps dans les échanges avec l'Empire ottoman comme les Français, Marseillais pour l'essentiel, et les Vénitiens. Si les marchands de la Sérénissime sont désormais loin de leur lustre d'antan, les Marseillais ont au contraire renforcé leurs positions en 1669 , avec l'obtention d'une franchise et de privilèges fiscaux pour leur port. Des marchands d'un autre port sont apparus au XvII ${ }^{\mathrm{e}}$ siècle, ceux de Livourne. Avec les Leggi Livornine (1590-1603), le port toscan abrite un ensemble hétéroclite de négociants engagés dans le commerce levantin. La présence de ces Anglais, Hollandais, Arméniens, Juifs ou Turcs est le fruit de la politique des Médicis qui a fait de Livourne un port franc, une terre d'accueil pour les marchands étrangers. La cité toscane est l'exemple le plus achevé d'une réalité touchant les grands ports de l'Europe méditerranéenne jusqu'à l'arrivée d'un nouveau pic d'intransigeances religieuses en Espagne et en France dans les années $1680^{27}$. Parler des négociants des villes portuaires italiennes, espagnoles et françaises, c'est certes évoquer des dynamismes purement locaux mais c'est aussi faire référence à des apports extérieurs, ceux des communautés étrangères, souvent non catholiques et parfois orientales.

Le commerce textile intra-méditerranéen est dans une large mesure une affaire communautaire. Et nous retrouvons ici les Arméniens. Ils exercent en Europe les activités pratiquées en Asie et tirent donc profit du commerce des indiennes. La présence de colonies facilite leur négoce. Chaque grand port de Méditerranée abrite un groupe de 
compatriotes favorisant le travail des agents itinérants faisant le voyage depuis l'Orient avec leurs balles de soies et de cotonnades. Les marchands arméniens installés dans ces ports ont misé sur la redistribution de ces indiennes mais en font aussi le commerce direct depuis les Échelles du Levant. Quelques personnalités marquantes émergent de ce groupe comme Antonio Boghos Chelebi, ancien douanier de Smyrne et négociantarmateur de Livourne dans les années 1660-1670, ou Melchion de Nazar, né à la NouvelleDjoulfa et «consul de la nation arménienne » à Marseille dans les années 1670-1690. Ces marchands arméniens sont-ils présents dans tous les circuits de distribution en Méditerranée? Rares sont ceux où ils pèsent peu. Il en existe toutefois. Ainsi, les exportations d'indiennes vers les États barbaresques sont entre les mains des marchands juifs, à l'image de Joseph Vaiz Villareal et d'Abraham Attias, beau-père et gendre associés et installés à Marseille puis à Livourne. Ces négociants s'appuient sur la diaspora séfarade pour alimenter Alger, Tunis et Tripoli en cotonnades ottomanes ou indiennes ${ }^{28}$.

\section{Ports majeurs et secondaires}

Dans la seconde moitié du XVII ${ }^{e}$ siècle, le commerce de gros des indiennes en Europe méditerranéenne s'appuie, en même temps qu'il la dessine, sur une hiérarchie portuaire dominée par Marseille et Livourne. L'échelle d'Alep, la principale du Levant avec Smyrne, permet de mesurer l'importance des deux villes pour l'accueil des cotonnades asiatiques. En 1686, les marchands marseillais et livournais prennent en charge l'expédition de 18.650 pièces depuis Alexandrette et totalisent donc plus des trois quarts $(78,2 \%)$ des envois vers l'Europe ${ }^{29}$. Les deux ports jouent autant la concurrence que la complémentarité car ils restent en relations constantes pour la gestion de leurs stocks de toiles et la bonne composition des assortiments. Selon les arrivages et les besoins du moment, les indiennes voyagent entre Livourne et Marseille. Les activités de Villareal \& Attias sont ici exemplaires. Installés à Marseille, ils expédient régulièrement des indiennes vers Livourne dans les années $1670^{30}$. En 1685, désormais installés à Livourne, après leur expulsion de France, ils ont un chargement de plus de 250 cotonnades destinées à la vente dans un magasin marseillais ${ }^{31}$.

Arrivées à Marseille et à Livourne, nombreuses sont les indiennes à repartir vers d'autres directions méditerranéennes, et tout d'abord vers les ports italiens. Si Gênes reçoit la visite de quelques navires anglais et possède des relations directes avec les Echelles du Levant, les cotonnades asiatiques qu'elle accueille viennent d'abord de Livourne. Marseille ne néglige pas d'y envoyer quelques balles et ballots par l'intermédiaire des négociants français mais aussi juifs et arméniens ${ }^{32}$. Naples et Rome fonctionnent à l'identique et les documents mentionnant les trafics de cotonnades depuis la Provence et la Toscane vers ces deux villes abondent dans les archives.

20 La redistribution des indiennes par Marseille et Livourne touche aussi l'Espagne méditerranéenne. Les vaisseaux anglais et hollandais en provenance du Nord de l'Europe s'arrêtent rarement dans les ports de Malaga et d'Alicante, et encore moins dans celui de Barcelone $^{33}$. Les quantités de cotonnades expédiées par l'IEC ou la VOC depuis Londres et Amsterdam sont donc très médiocres ${ }^{34}$. Du côté des Échelles du Levant, le problème de l'accès direct des Espagnols aux indiennes par Smyrne et Alep se pose également. Les temps médiévaux des relations actives entre Barcelone et l'Orient sont lointains et les statistiques dressées par Eloy Martín Corrales le démontrent sans équivoque: pour l'ensemble du XVII ${ }^{e}$ siècle, neuf navires quittent les Échelles du Levant pour la Catalogne, 
dont un seul après $1633^{35}$. Les indiennes ont pourtant commencé à trouver leur voie vers l'Espagne. Les données collectées dans les archives des douanes catalanes permettent d'observer une augmentation de leur consommation au cours du XVII ${ }^{e}$ siècle ${ }^{36}$. Cette progression reste néanmoins lente et la présence des cotonnades dans les boutiques catalanes n'est ni massive ni populaire, comme les études de Lídia Torra Fernández l'ont démontré37. Longtemps, la toile asiatique en Catalogne est la blavet, la toile ottomane teinte en bleu indigo, et L'accélération décisive n'intervient qu'à l'extrême fin du siècle, notamment à Barcelone. La capitale catalane reçoit durant les années 1695-1696 pour 17.000 canas (environ 27.000 mètres) d'indiennes ${ }^{38}$. Dans les exportations vers Barcelone et les autres ports catalans, Livourne et surtout Marseille se distinguent encore ${ }^{39}$. Ce commerce est même vital pour les marchands de ces deux villes car il leur permet de recevoir en paiement les pièces d'argent nécessaires aux échanges avec le Levant.

\section{Contours de consommations}

21 À quoi servent ces indiennes en Méditerranée? Quels groupes sociaux en font la consommation? Peut-on déterminer les goûts des acheteurs? Le tableau présenté ici s'appuie sur l'exemple marseillais, le seul ayant fait l'objet de recherches suffisantes pour offrir des éléments de réponse.

Depuis le $\mathrm{XVI}^{\mathrm{e}}$ siècle et jusqu'aux années 1660 , les indiennes sont surtout utilisées dans la décoration des intérieurs. Tapis, tentures, rideaux, couvertures de table et de lit...: les usages des cotonnades dans l'ameublement sont multiples et partagés par l'ensemble de l'espace européen. A Marseille, les indiennes pour la décoration des intérieurs sont déjà présentes dans plus de la moitié des inventaires après-décès des années 1667-1668 et dans presque les trois quarts de ceux des années $1692-1693^{40}$. Les cotonnades pour l'habillement font l'objet d'une croissance plus marquée. À peine 5,1\% des inventaires marseillais contenaient des vêtements en indiennes à la fin des années 1660 . Ce pourcentage est multiplié par huit en 25 années seulement. À l'image de ce qui se produit au même moment en Angleterre ${ }^{41}$, les années 1670-1680 constituent un tournant. Les indiennes investissent le champ de l'habillement, garnissent les vestiaires et finissent même par quitter les intérieurs. La liste des nouveaux usages est déjà bien fournie dans la Marseille des années 1667-1692 : robes, manteaux, jupons, tabliers, chemises, mouchoirs, cravates et un vêtement emblématique de l'époque, porté par les hommes comme par les femmes, la robe de chambre, celle du bourgeois gentilhomme de Molière, appelée en France "robe d'Arménien ", soulignant une nouvelle fois le rôle de cette communauté dans le commerce des cotonnades asiatiques. Parmi les 37 détenteurs identifiés de robes de chambre, nous trouvons des consommateurs issus des catégories supérieures de la société phocéenne (sept marchands, trois capitaines de navires, un bourgeois, un avocat, un notaire...) mais aussi de nombreux représentants du monde de la boutique et de l'artisanat, voire de certaines couches populaires (deux meuniers, deux maçons, un fabricant de savon, un charcutier, deux marins, deux pêcheurs, un tanneur, un passementier...). Cette tendance de démocratisation de la consommation des indiennes se retrouve-t-elle partout en Européenne méditerranéenne? Il parait logique de le penser mais la rapidité et l'intensité du phénomène ont certainement été différentes selon les régions.

Peut-on se faire une idée des goûts des consommateurs? Pour ce qui touche aux couleurs, les renseignements sont peu abondants mais existent néanmoins. Nous l'avons vu plus 
haut, les véritables indiennes n'avaient pas la préférence des Européens. Ceci était particulièrement vrai pour les cotonnades du Nord-Ouest de l'Inde dont les fonds en rouge et en violet et les petites fleurs éparpillés en négatif étaient à l'opposé de ce qui était recherché. Les Européens affectionnaient les indiennes à dessins soignés sur fond blanc comme l'atteste la correspondance de la maison de commerce marseillaise Tiran \& Rampal pour les années $1679-1684^{42}$. Cette importance des fonds blancs se retrouve dans les couvertures de lit en indiennes présentes dans les inventaires après-décès phocéens. Ainsi, en 1692, on observe que plus des deux tiers de celles dont nous connaissons la couleur sont à fond blanc (19 sur 28) et le reste à fonds colorés. La couleur des motifs est rarement mentionnée et, quand c'est le cas, il s'agit surtout de fleurs rouges. Les Marseillais affectionnent avant tout les motifs floraux naturalistes et les dessins assez grands et assez fins. Pour les indiennes, les sociétés méditerranéennes possèdent globalement les mêmes goûts que les sociétés du Nord de l'Europe.

\section{Les débuts de l'indiennage en Europe méditerranéenne}

Le dynamisme et l'habileté des compagnies et des marchands engagés dans le commerce eurasiatique ne sont pas seuls responsables de la diffusion des indiennes en Europe. Dès le milieu du XvII ${ }^{\mathrm{e}}$ siècle, des productions d'imitation sont lancées sur le vieux continent. Les villes portuaires de la Méditerranée jouent un rôle pionnier dans les débuts de ce processus de substitution aux importations orientales. Avec l'introduction de cette fabrication, les populations européennes finissent de s'approprier les indiennes, commencent à devenir autonomes dans la satisfaction de leurs besoins et avancent dans la maîtrise des techniques asiatiques.

\section{L'Europe à l'épreuve du défi technologique asiatique}

Entre 1648 et 1678, les premières fabriques d'indiennes apparaissent à Marseille, Avignon, Gênes, Rome, Paris, Londres et Amsterdam. Les débuts sont difficiles car les couleurs apposées sur les tissus manquent de solidité. Les artisans européens travaillent en « petit teint ». Ils peinent à percer par eux-mêmes les secrets orientaux de la préparation et de l'application des mordants pour la coloration, notamment pour le rouge garance ${ }^{43}$. Ainsi, l'atelier ouvert par William Sherwin en 1676 à West Ham, le premier d'Angleterre, produit pendant longtemps des indiennes dont les motifs s'effacent avec les lavages ${ }^{44}$. En Méditerranée, le constat est identique: les premières fabrications de Marseille et de Gênes ne rivalisent pas avec les productions asiatiques ${ }^{45}$. Ce n'est qu'à partir des années 1670-1690 que l'Europe se dote des savoir-faire nécessaires pour fabriquer en «bon teint». Comment ces techniques ont-elles cheminé depuis l'Inde? Les voyageurs européens en Asie ont été très tôt fascinés par l'indiennage. Dès 1547, l'apothicaire Pierre Belon du Mans décrit les méthodes d'impression sur coton dans l'Empire ottoman lors d'un voyage à Constantinople. Au cours du XVII siècle, les observations européennes se font plus précises et concernent désormais l'Inde. Le phénomène culmine dans les années 1678-1688 avec les récits d'agents des compagnies des Indes comme celui du Français Georges Roques pour le Gujarat et ceux des Hollandais Daniel Havart et Hendrick Van Rheede pour la côte de Coromandel. L'impact reste insignifiant pour l'industrie européenne. Dans une époque où les connaissances sont encore empiriques, l'écrit 
compte peu. Les techniques ne voyagent qu'avec les artisans qui les maîtrisent et l'apprentissage par la pratique en atelier s'impose.

La route transocéanique ne semble pas avoir compté dans les transferts des technologies orientales de l'indiennage. Tout démontre en revanche que les routes caravanières intraasiatiques ont eu un rôle déterminant. C'est vers et depuis les Echelles du Levant que les savoir-faire indiens ont progressé d'est en ouest et se sont étoffés et transformés chemin faisant. Le vieux continent n'a pu trouver en son sein les hommes pour opérer ce transfert et ce sont donc des Orientaux qui ont assuré la médiation technique. Ceux-ci étaient Arméniens et il n'y a là rien de surprenant. Ils étaient impliqués dans le commerce des indiennes mais avaient également investi le secteur de l'impression sur coton. Avant de traverser la Méditerranée, les Arméniens avaient déjà pris en charge le transfert des techniques de l'indiennage à travers le continent asiatique et peuplaient les ateliers d'Ispahan, de Smyrne, d'Alep et de Constantinople. Compétents techniquement, chrétiens et donc susceptibles de mieux s'intégrer dans les tissus économiques et sociaux du vieux continent, ils étaient les hommes idoines pour apporter aux Européens les compétences qui leur faisaient défaut.

\section{L'apport des Arméniens à l'indiennage méditerranéen}

Les indienneurs arméniens de l'Empire ottoman et de Perse traversent la Méditerranée à partir du début des années 1670. Les premiers sont à l'œuvre à Marseille où trois d'entre eux (Boudac, Dominique Ellia et Arapié d'Arachel) s'associent pour fabriquer à la façon «du Levant et de Perse $~_{46}$. D'autres suivront rapidement et, grâce aux Arméniens, la cité phocéenne maitrise les techniques de production des indiennes en moins de dix ans. Les recherches menées en Italie laissent à penser qu'ils ont joué un rôle identique dans la République de Gênes et dans le Grand-Duché de Toscane. A Gênes, l'indiennage s'affirme dans les années 1670 avec la présence d'une fabrique peut-être entre les mains de deux marchands arméniens, les frères Arattone et Giovanni di Arachelle ${ }^{47}$. En 1690, le poids des producteurs arméniens y est clairement attesté puisque Giobatta de Georgiis, « arménien de nation", reçoit des gouverneurs génois un monopole exclusif de dix ans pour l'impression des «fines couleurs de Perse » et «teindre les toiles rouges du Levant " ${ }^{48}$. D'après Frédéric Macler, les Arméniens ont également appris aux Livournais l'art de l'impression sur coton ${ }^{49}$.

La diffusion des techniques de l'indiennage par les Arméniens se fait aussi indirectement. Les artisans européens formés par les Arméniens à Marseille ou en Italie ont été mobiles et ont participé à la diffusion de ces savoir-faire. Ainsi, Antoine et Clément Barges, frères et marchands associés, engagent Vincent Mille "peintre d'indiennes dudit Marseille", ancien ouvrier dans un atelier tenu par un Arménien, pour aller travailler pour eux à Rome en $1677^{50}$. Un peu plus tard, après 1689 et l'application de la prohibition des indiennes à Marseille, les indienneurs marseillais partent pour la Toscane, où le GrandDuc «leur donne beaucoup de protection $»^{51}$. A n'en pas douter, certains d'entre eux avaient côtoyé des Arméniens ou avaient été formés par ces derniers. La fondation de la première fabrique hollandaise en 1678 permet également d'observer la présence d'ouvriers arméniens et le rôle de Marseille dans les débuts de l'impression sur coton en Europe du nord. La direction technique de la production y est en effet confiée à «monsieur Louis de Çelebi», un indienneur arménien sans doute passé par la cité phocéenne auparavant ${ }^{52}$. 


\section{Le retard catalan : une affaire de marchés?} la Catalogne se présente comme un cas intéressant car la mention la plus ancienne de cette activité est tardive. Elle date seulement de 1712 et la première véritable fabrique n'y est fondée qu'en $1736^{53}$. Pourquoi ce retard? Faut-il l'expliquer par une incapacité technologique ou un manque de capitaux ? La réponse est négative sur les deux points. Même si les Catalans ne disposaient pas des compétences techniques nécessaires dans les années 1670-1680, ils pouvaient toujours les transférer et ce sera d'ailleurs le cas dans les années 1730, quand les premiers fabricants se tourneront vers les Marseillais ${ }^{54}$. Le problème des capitaux ne se posait pas non plus quand on sait que l'investissement nécessaire à l'entrée dans l'industrie, et notamment dans l'indiennage, était faible à cette époque. Reste la piste des marchés.

La date de 1712 suit de peu le décollage de l'importation des indiennes en Catalogne. Le marché pourrait donc être la cause de ce retard et l'histoire européenne semble accréditer cette thèse. Au XVII siècle, Barcelone et sa région ont été durement touchées par une longue $\mathrm{crise}^{55}$. La reprise tarde un peu plus longtemps que dans certaines régions italiennes ou françaises et il faut attendre les années 1680 pour qu'elle se manifeste. C'est cette reprise qui aurait lancé les importations d'indiennes puis les premières tentatives de substitution aux produits asiatiques. La Catalogne serait entrée avec retard dans cette « révolution industrieuse » qui touche l'Europe de la seconde moitié du XvII ${ }^{\mathrm{e}}$ siècle. Mise en lumière par l'historien Jan de Vries ${ }^{56}$, cette révolution a accompagné le développement de la consommation de produits manufacturés, dont les indiennes, dans plusieurs régions européennes. Elle s'appuie sur une transformation des comportements des populations, qui consentent alors à réduire leur temps de repos et à profiter de leur temps mort afin de fournir un effort pour la production de biens destinés au marché. Leur objectif ? L'augmentation de leurs revenus monétaires, afin d'accroître et de diversifier leur consommation. Ce n'est qu'à partir des années 1680, avec le développement manufacturier et artisanal de la région, que les familles catalanes auraient modifié leur comportement au travail, changement qui aurait porté ses fruits au début du XVIII ${ }^{\mathrm{e}}$ siècle. Cette hypothèse reste encore à être validée et il faudrait pour cela pouvoir disposer d'une étude précise des inventaires après-décès catalans entre 1650 et 1720 . Ceux-ci attendent encore leur historien.

\section{ANNEXES}

Tab. 1 : nomenclature des cotonnades asiatiques présentes dans l'inventaire après-décès d'Arattone di Arachelle, marchand arménien de Gênes (1678)

\begin{tabular}{|l|l|}
\hline Pièces brutes : scaparoni et ritagli & Lieux de fabrication
\end{tabular}




\begin{tabular}{|l|l|}
\hline Casses, mousselines et percales & Inde \\
\hline Demittes blanches et couleur argent ; escamittes blanches et turques & Empire ottoman \\
\hline Indiennes turques et de Smyrne & Empire ottoman \\
\hline Indiennes de Perse & Perse et Inde \\
\hline Pièces taillées : coperte, fazzoletti, mezzari, robbe da camera... & Lieux de fabrication \\
\hline Couvertures de Brousse ; d'Alep et de Brousse & Empire ottoman \\
\hline Voiles de Sironj (serongis) & Inde \\
\hline Mouchoirs d'Alep et de Brousse & Empire ottoman \\
\hline Mouchoirs blancs travaillés à la turque & Empire ottoman et Inde? \\
\hline Mouchoirs d'indiennes & Inde ou Inde et Perse \\
\hline Robes de chambre pour enfants et pour adultes (hommes et femmes) & Inde ou Inde et Perse \\
\hline
\end{tabular}

Source : Margherita BELLEZzA RosinA, Marzia CATALDI GALlo, Cotoni stampati e mezzari dalle indie all'Europa, Gênes, Sagep, 1993, p. 72-73.

Tab. 2 : cotonnades asiatiques présentes dans l'inventaire de la boutique marseillaise de Jeanne Montagne (1683)

\begin{tabular}{|l|l|}
\hline Quantités et types de pièces & Provenances \\
\hline 20 grandes couvertures de Perse & Perse et Inde \\
\hline 19 robes de chambre pour hommes et femmes sur calankars & Inde \\
\hline 8 couvertures guinées blanches & Inde \\
\hline 5 lisats fines & Inde \\
\hline 607 indiennes d'Ispahan & Perse et Inde \\
\hline 68 calankars de diverses qualités & Inde \\
\hline 9 robes de chambre sur indiennes demi calankars & Inde \\
\hline 6 lisats ordinaires & Inde \\
\hline 3 couvertures sur boucassin avec broderies & Empire ottoman \\
\hline 14 couvertures du Levant & Empire ottoman \\
\hline 99 indiennes de Sironj & Inde \\
\hline
\end{tabular}




\begin{tabular}{|l|l|}
\hline 153 indiennes d'Ispahan à fond noir & Perse \\
\hline 41 robes de chambre d'été pour hommes et femmes & Inde et/ou Perse \\
\hline 10 lisats imprimées à Smyrne & Empire ottoman et Inde \\
\hline 27 indiennes d'Ispahan ordinaires & Perse ou Perse et Inde \\
\hline 80 robes de chambre communes sur boucassins & Empire ottoman \\
\hline 66 indiennes varas fines & Inde \\
\hline 350 indiennes varas & Inde \\
\hline 22 indiennes chafarcanis & Inde \\
\hline 276 indiennes grossières & $?$ \\
\hline 220 pièces boucassin « à double teinture » & Empire ottoman \\
\hline 415 boucassins à une couleur & Empire ottoman \\
\hline
\end{tabular}

Source : Arch. dép. BdR, 355 E 454, fol. 360 vº 23 août 1683.

Tab. 3 : indiennes exportées vers l'Europe par les compagnies des Indes (1665-1684) (moyennes annuelles)

\begin{tabular}{|l|l|l|l|l|}
\hline & Angleterre & Hollande & France & Totaux \\
\hline $1665-1669$ & 139.700 & 126.600 & - & 265.700 \\
\hline $1670-1674$ & 510.500 & 257.900 & & 808.400 \\
\cline { 1 - 3 } $1675-1679$ & 580.900 & 127.500 & $50.000 *$ & 758.400 \\
\cline { 5 - 6 } $1680-1684$ & 973.800 & 226.800 & $100.000 *$ & 1.299 .800 \\
\hline
\end{tabular}

* : moyennes annuelles pour la période.

Sources : Kirti Narayan CHAUDHURI, The Trading World of Asia and the English East India Company, 1660-1760, Cambridge, CUP, 1978, p. 540-541; Femme Simon GAASTRA, « The Textile Trade of the VOC », South Asia, $n^{\circ}$ 19, 1996, p. 85-95 ; Philippe HAUDRÈRE, « La Compagnie des Indes et le commerce des toiles indiennes » in Jacqueline Jacqué, Brigitte NiColas (dir.), Féérie indienne. Des rivages de l'Inde au royaume de France, Paris, Somogy, 2008, p. 16.

Tab. 4 : les exportations vers l'Europe de la VOC et de l'EIC (en \% des valeurs totales)

\begin{tabular}{|l|l|l|l|l|}
\hline & $1619-1621$ & $1648-1650$ & $1668-1670$ & $1698-1700$ \\
\hline
\end{tabular}




\begin{tabular}{|l|l|l|l|l|l|l|l|l|}
\hline & VOC & EIC & VOC. & EIC. & VOC & EIC & VOC & EIC \\
\hline Epices & 74,1 & $?$ & 68,3 & $?$ & 42,6 & 25,25 & 22,9 & 7,02 \\
\hline Textiles & 16,1 & $?$ & 14,2 & $?$ & 36,5 & 56,61 & 54,7 & 73,98 \\
\hline
\end{tabular}

Source : Ronald Finlay, Kevin H. o'Rourke, Power and Plenty: Trade, War and the World Economy in the Second Millenium, Princeton, PUP, 2007, p. 308-309.

Tab. 5 : exportations d'indiennes par Alep en 1686 (en pièces)

\begin{tabular}{|c|c|c|c|c|c|}
\hline Produits & Français & Anglais & Hollandais & Vénitiens & Livournais \\
\hline Indiennes d'Alep & 300 & - & - & 400 & 2.000 \\
\hline Mouchoirs d'Alep (12 par pièce) & 1.000 & - & 1.000 & - & 1.000 \\
\hline \multicolumn{6}{|l|}{ Indiennes perses } \\
\hline Indiennes d'Ispahan & 1.500 & 1.000 & 3.000 & - & - \\
\hline \multicolumn{6}{|l|}{ Indiennes des Indes en pièces longues } \\
\hline Indiennes « calanca » & 200 & 200 & - & - & 100 \\
\hline Indiennes « serongis" & 2.000 & - & 500 & - & 1.000 \\
\hline Indiennes « chefercany » grandes & 600 & - & - & - & 500 \\
\hline Indiennes « chefercany » petites & 400 & - & - & - & 500 \\
\hline Indiennes « vara » & 500 & - & - & - & - \\
\hline Tapis « calanca » & 100 & 100 & - & - & 100 \\
\hline Tapis « demi-calanca » & 50 & - & - & - & 200 \\
\hline Tapis « serongis» & 600 & - & - & - & 600 \\
\hline Tapis de lit & 100 & - & - & - & 300 \\
\hline \multicolumn{6}{|l|}{ Mouchoirs en indiennes des Indes } \\
\hline Mouchoirs « cassary » (15 par pièce) & 100 & - & - & - & 100 \\
\hline Mouchoirs « chevely » ( 3 par pièce) & 700 & - & - & - & 100 \\
\hline Mouchoirs ordinaires (12 par pièce) & 2.000 & - & - & - & 1.000 \\
\hline Totaux & 10.150 & 1.300 & 4.500 & 400 & 7.500 \\
\hline
\end{tabular}


Source : BNF, ms fr 7174 , fol. 447 et suiv.

Tab. 6 : les indiennes dans les inventaires après décès marseillais (1667-1693)

\begin{tabular}{|l|l|l|l|l|l|}
\hline & $\begin{array}{l}\text { Nombre } \\
\text { d'inventaires }\end{array}$ & $\begin{array}{l}\text { Avec indiennes } \\
\text { d'ameublement }\end{array}$ & $\begin{array}{l}\% \quad \text { par } \\
\text { rapport au } \\
\text { total }\end{array}$ & $\begin{array}{l}\text { Avec indiennes } \\
\text { pour l'habillement }\end{array}$ & $\begin{array}{l}\text { par } \\
\text { rapport au } \\
\text { total }\end{array}$ \\
\hline $1667-1668$ & 135 & 70 & 51,85 & 7 & 5,19 \\
\hline $1680-1681$ & 112 & 77 & 68,75 & 27 & 24,11 \\
\hline $1692-1693$ & 178 & 133 & 74,72 & 74 & 41,58 \\
\hline
\end{tabular}

Source : Arch. dép. BdR, 2 B (1/1/1667 au 17/2/1667 et 11/11/1667 au 31/12/1668 ;

$1 / 1 / 1680$ au $4 / 3 / 1681 ; 1 / 1 / 1692$ au $1 / 8 / 1693)$.

Carte 1 : Les principaux centres textiles indiens (XVIIe-XVIII siècles)

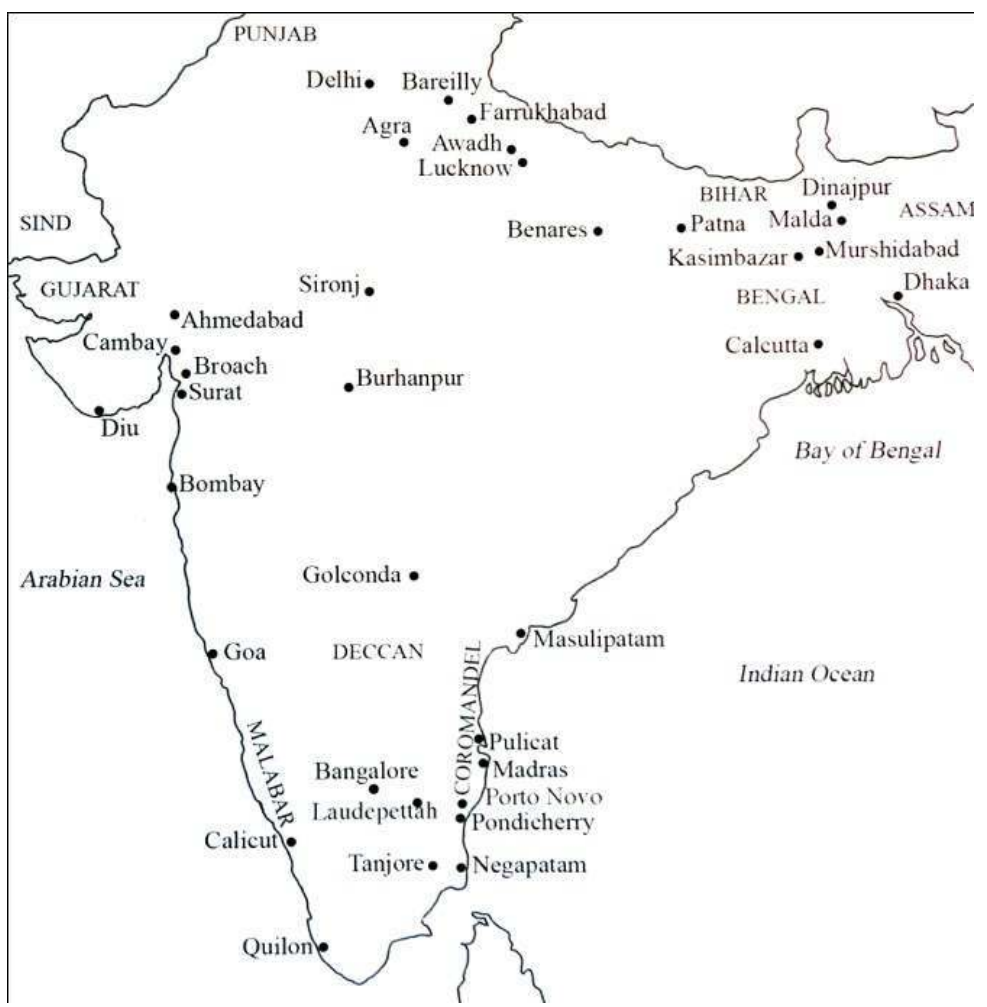

Source: Prasannan PARTHASARATHI, « Cotton Textiles in the Indian Subcontinent, 1200-1800 » in Giorgio RIELlo, Prasannan PARTHASARATHI (eds), The Spinning World..., op. cit., p. 19). 
Carte 2 : Les routes commerciales intra-asiatiques au XVIIe siècle

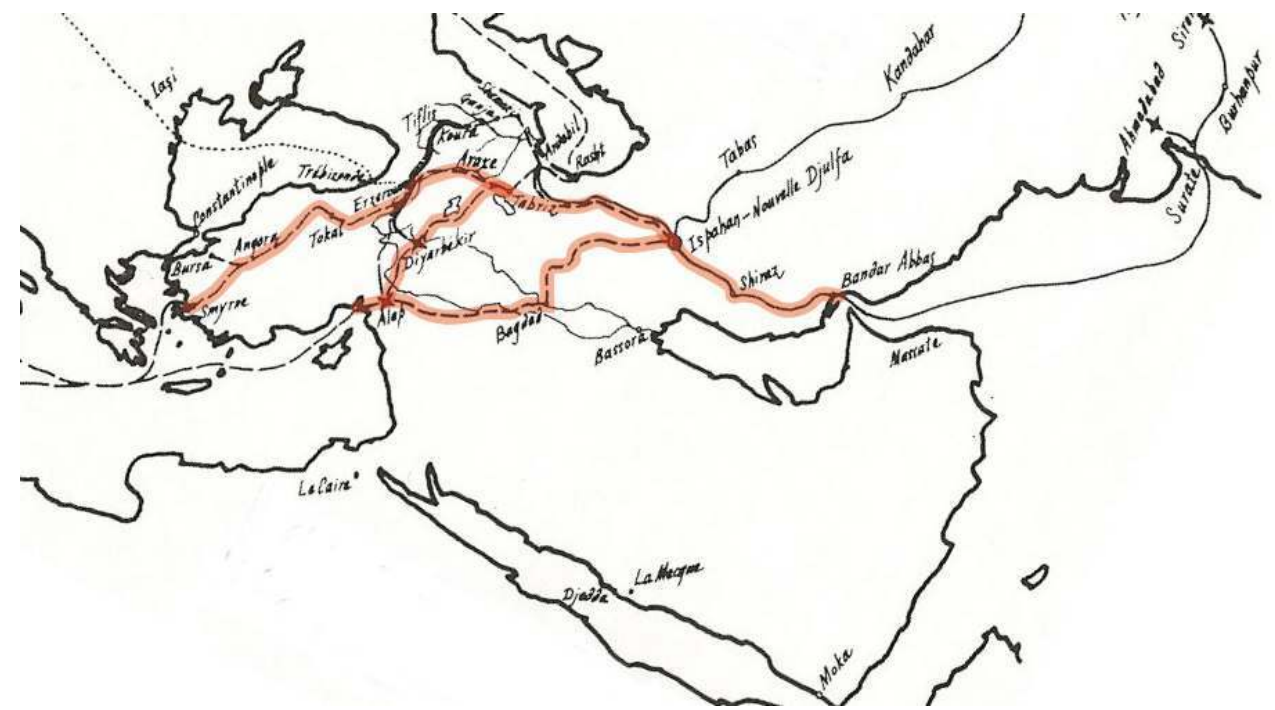

Source : Katsumi FUKASAWA, Toilerie et commerce du Levant d'Alep à Marseille, Marseille, CNRS, 1987, p. 27.

\section{NOTES}

1. Patrick VERLEY, L'Échelle du monde. Essai sur l'industrialisation de l'Occident, Paris, Gallimard, 1997, 718 p. et Giorgio Riello, Prasannan Parthasarathi (eds), The Spinning World: A Global History of Cotton Textile, 1200-1850, Oxford, OUP, 2009, 506 p.

2. Cf. tableau 3 (cartes et tableaux en fin d'article). Une pièce fait une dizaine de mètres de longueur en moyenne.

3. Ralph DAVIS, « English Foreign Trade, 1660-1700 », Economic History Review, 1954/2, p. 150-166.

4. Cf. tableaux 1 et 2 .

5. Cf. carte 1 et Prasannan PARTHASARATHI, "Cotton Textiles in the Indian Subcontinent, 1200-1800 » in Giorgio RIello, Prasannan Parthasarathi (eds), The Spinning World..., op. cit., p. $18-41$.

6. Katsumi FUKASAWA, Toilerie et commerce du Levant d'Alep à Marseille, Marseille, CNRS, 1987, p. 37.

7. Cf. carte 2 .

8. Sur les indiennes de Perse: Willem FLooR, «The import of Indian textiles into Safavid Persia », Eurasian Studies, V/1-2, 2006, p. 107-141.

9. Bibliothèque Nationale de France (désormais BNF), FOL- FM- 15857 (2), Rapport du 26 juillet 1675.

10. $C f$. tableau 2.

11. Sebouh-David ASLANIAN, From the Indian Ocean to the Mediterranean. The Global Trade Networks of Armenian Merchants from New Julfa, Berkeley, UCP, 2011, 363 p.

12. BNF, ms fr. 7.174, fol. 460 et ms fr. 14.614, fol. 16 .

13. Le prix des épices n'augmente plus à partir de 1680 (Philippe HAUDRÈrE, « Naissance du goût de l'Inde en Europe » in Gérard LE BOUËDEC, Brigitte NICOLAs (dir.), Le Goût de l'Inde, Rennes, PUR, 2008, p. 11).

14. Cf. tableau 4 . 
15. John IRWIN, « Origins of the 'Oriental Style' in English Decorative Art », The Burlington Magazine , $\mathrm{n}^{\circ} 625,1955$, p. 109; «Indian Textile trade in the Seventeenth Century: IV Foreign Influences », Journal of Indian Textile History, IV, 1959, p. 57.

16. John STYLES, «Product Innovation in Early Modern London », Past and Present, $n^{\circ} 168,2000$, p. 133; D. A. Farnie, «The Role of Cotton Textiles in the Economic Development of India, 1660-1990 " in The Fibre that Changed the World. The Cotton Industry in International Perspectives, 1600-1990s, Oxford, OUP, 2004, p. 397.

17. BNF, ms fr. 7.174, « Mémoire de l'état présent du négoce de Smyrne » (1688).

18. Charles CAPPER, The Port and Trade of London, London, 1862, p. 81-82.

19. Jacques SAVARY DES BRUSLONS, Dictionnaire universel de commerce, Paris, t. I, 1741, p. 200-220.

20. L'expression est de John Fitch, agent diplomatique à Livourne en 1667 (cité dans Michela D’ANGELO, « Mercanti inglesi a Livorno (1573-1796) » in Adriano PROSPERI (dir.), Livorno, 1606-1806. Luogo di incontro tra popoli e culture, Torino, Allemandi, 2009, p. 354-355).

21. William FOSTER, Ethel Bruce SAINSBURY, A Calendar of the Court Minutes of the East India Company, 1660-1663, Oxford, 1922, p. 262.

22. Id., années 1671-1679 (Oxford, 1938, p. 321) ; Archives départementales des Bouches-du-Rhône (désormais Arch. dép. BdR), 2 E 17, testament de Robert Lang.

23. Arch. dép. BdR, 394 E 27, 21 janvier 1677, fol. $62 \mathrm{v}^{\circ}$.

24. Arch. dép. BdR, 394 E 35, 10 janvier 1685, fol. 26 et 394 E 39, $1^{\text {er }}$ mars 1689, fol. 142.

25. Cf. Antonella Bicci, «Gli Olandesi nel Mediterraneo: Amsterdam e l'Italia (sec. XVII)» in Economies méditerranéennes. Équilibres et intercommunications, Athènes, FNRS, 1985, p. 39-76.

26. Cf. tableau 5.

27. En 1683, les Arméniens sont expulsés du royaume d'Espagne (Antonio DOMÍNGUEZ ORTIZ, Los extranjeros en la vida española durante el siglo XVII y otros artículos, Sevilla, 1996, p. 121). Louis XIV expulse les Juifs de son royaume la même année et interdit aux Arméniens le négoce de la soie en France en 1687.

28. Les polices de chargement de Villareal \& Attias entre 1670 et 1679 permet de saisir l'ampleur de ce commerce (Arch. dép. BdR, 394 E 29).

29. Cf. tableau 5 .

30. $C f$. note 30 .

31. Arch. dép. BdR, 394 E 35.19 novembre 1685, fol. $801 \mathrm{v}^{\circ}$.

32. Archives de la Chambre de Commerce de Marseille, C 123, police du 5 octobre 1691.

33. Gigliola PAGANO DE DIVITIIS, Mercanti inglesi nell'Italia del Seicento, navi, traffici, egemonie, Venise Marsilio editori, 1990, $223 \mathrm{p}$..

34. Juan Antonio SÁNCHEZ BELÉN, «El comercio de exportación holandés en el Mediterráneo español durante la regencia de doña Mariana de Austria ", Espacio, Tiempo y Forma, IV/9, 1996, p. 267-361.

35. Eloy martín corrales, Comercio de Cataluña con el Mediterráneo musulmán (siglos XVI-XVIII). El comercio con los « enemigos de la fe », Barcelona, Bellaterra, 2000, p. 602 et suivantes.

36. Eloy MARTín CORRALES, «La importación de telas de algodón levantino y los inicios del estampado en Cataluña », Revista de Historia Industrial, $n^{\circ}$ 6, 1994, p. 47-74.

37. Lidia TORRA FERNÁNDEZ, "Cambios en la oferta y la demanda textil en Barcelona (1650-1800) », Revista de Historia Industrial, $\mathrm{n}^{\circ} 22,2002$, p. 16).

38. José FONTANA LÁZARO, «Sobre el comercio exterior de Barcelona en la segunda mitad del siglo XVII », Estudios de Historia Moderna, vol. nº 5, 1955, p. 210.

39. Eloy mARTín CORRALES, "Marseille, échelle des toiles levantines pour l'Espagne aux XVII et XVIII ${ }^{\mathrm{e}}$ siècles », Rives Nord-Méditerranéennes, $\mathrm{n}^{\circ}$ 29, 2008, p. 61-78.

40. Cf. tableau 6. 
41. Beverly LEMIRE, « Fashioning Cottons: Asian trade, domestic industry and consumer demand 1660-1780 » in David JENKIns (ed.), The Cambridge History of Western Textiles, Cambridge, CUP, 2003, vol. 2, p. 493-512.

42. Arch. Dép.BdR, 9 B 175, Registre de correspondance Tiran \& Rampal, Smyrne, 1679-1984.

43. Ernst HOMBURG, « From Colour Maker to Chemist: Episodes from the Rise of the Colourist » in Robert FoX, Augusti NIETo GÁLAN (dir.), Natural Dyestuffs and Industrial Culture in Europe, 1750-1880, Canton, 1999, p. 221.

44. Parakunnel Joseph тномаs, "The Beginnings of Calico-Printing in England », English Historical Review, 1924, vol. 39/154, p. 209.

45. Pour ce paragraphe, $c f$. Giorgio RIELLo, "Asian Knowledge and the Development of Calico Printing in Europe in the Seventeenth and Eighteenth Centuries ", Journal of Global History, 2010/5, p. 1-28.

46. Olivier RAVEuX, «À la façon du Levant et de Perse : Marseille et la naissance de l'indiennage européen (1648-1689) », Rives Nord-Méditerranéennes, $\mathrm{n}^{\circ}$ 29, 2008, p. 37-51.

47. Margherita BELLEZZA Rosina, Marzia CATALdi GALlo, Cotoni stampati e mezzari dalle Indie all'Europa , Gênes, Sagep, 1993, p. 73-74.

48. Orietta SPIRITO, Voiles de Gênes, Mulhouse, 1964, p. 4.

49. "Notes de Chahan de Cirbied sur les Arméniens d'Amsterdam et de Livourne », Anahit, 1904, p. 11.

50. Arch. dép BdR, 351 E 994.

51. François Xavier emmanuelLI, L'Intendance de Provence à la fin du XVII siècle: mémoires pour l'instruction du duc de Bourgogne, Paris, BNF, 1980, p. 172.

52. Ernst HOMBURG, « From colour... », art. cit., p. 222.

53. Cf. Eloy martín CORRALEs, "Marseille...», op. cit., p. 53 et James thOMSON, Els orígens de la industrialització a Catalunya. El cotó a Barcelona, 1728-1832, Barcelona, Edicions 62, 1994.

54. Agusti DURAN, Barcelona i la seva història. La societat i l'organització del treball, Barcelona, Curial, 1973, p. 292-293.

55. James tномSON, Els orígens..., op. cit., p. 52-57.

56. Jan DE VRIES, «The industrial revolution and the industrious revolution », Journal of Economic History, 54-2, 1994, p. 249-270.

\section{AUTEUR}

\section{OLIVIER RAVEUX}

Chargé de recherche CNRS, Laboratoire Temps, Espaces, Langages, Europe Méridionale Méditerranée (TELEMMe, UMR 7303), MMSH, 5, rue du Château de l'Horloge, BP 647, 13094 Aixen-Provence cedex 2 